\title{
The Missing Link between Ki-67 and Papillary Thyroid Carcinoma
}

\author{
Shahrun Niza Abdullah Suhaimi ${ }^{1}$, Mohamad Aznan Shuhaili², Nurismah Md Isa ${ }^{3}$, Suria Hayati Md Puad ${ }^{4}$, \\ Shamsul Azhar Shah ${ }^{5}$, Rohaizak Mohammad ${ }^{6}$
}

\begin{abstract}
Introduction: Ki-67 antigen was originally defined by the prototype monoclonal antibody Ki-67 detected by immunizing mice with nuclei of the Hodgkin lymphoma cell line L-428. ${ }^{2}$ The name derived from Kiel (city of origin) and the number of the original clone in the 96-well plate. $\mathrm{Ki}-67$ has been thoroughly investigated in cases of both benign and malignant thyroid nodules. However, few previous studies have focused on a potential link between prognostic factors of papillary thyroid carcinoma (PTC) and the Ki-67 proliferative index. The objective of this study was to determine the prognostic significance of Ki-67 levels and PTC. We compared Ki-67 levels against the metastasis, age, completeness of resection, invasion, and size (MACIS) tumor scoring system. By correlating Ki-67 with poor prognostic features of thyroid carcinoma, we aimed to predict tumor recurrence in PTC.

Materials and methods: A total of 46 PTC patients who had underwent surgery from 2006 to 2012 were involved in this study. All of the surgical specimens were analyzed for Ki-67 through immunohistochemistry (IHC), and two independent pathologists evaluated the Ki-67 staining results. We compared Ki-67 levels with various prognostic factors for PTC.

Results: There was no significant relationship between the Ki-67 index and age, tumor size, cervical lymph nodes involvement, or complete tumor removal during initial surgery ( $p$ value $>0.05$ ). However, there were significant links between Ki-67 levels and extrathyroidal extension ( $p$ value $=0.006)$, vascular invasion ( $p$ value $=0.006)$, and distant metastasis ( $p$ value $=0.005)$. Ki-67 was significantly reduced among the lowrisk group for recurrent PTC ( $p$ value $=0.007$ ). Tumor recurrence at 3 years was significantly correlated with high Ki-67 levels $(p$ value $=0.01$ ). Keywords: Ki-67, Papillary thyroid carcinoma, Prognostic factors.

World Journal of Endocrine Surgery (2020): 10.5005/jp-journals-10002-1287
\end{abstract}

\section{INTRODUCTION}

The Ki-67 antigen is strictly a marker for cell proliferation. It is detected through immunohistochemistry, where antibodies against the $\mathrm{Ki}-67$ protein are used. $\mathrm{Ki}-67$ is expressed in all stages of the cell cycle, except G0, and resting cells entering from G0 lack Ki-67 in the early phase of $\mathrm{G} 1 .^{1,2}$ Interestingly, tumors with a high concentration of Ki-67 literally translate into cancer. The usefulness of Ki-67 as a cancer marker has been well established for various types of malignancy, particularly multiple myeloma, soft tissue sarcoma, prostate carcinoma, and breast carcinoma. ${ }^{3-7} \mathrm{Ki}-67$ is generally low in thyroid carcinoma, except in anaplastic thyroid carcinoma and a poorly differentiated thyroid carcinoma. On the other hand, there are few studies reporting that Ki-67 antigen levels differ between benign and malignant nodules. ${ }^{8,9}$ In well-differentiated thyroid carcinomas, however, Ki-67 has been reported in medullary, follicular, and papillary thyroid carcinomas at low level. ${ }^{8-11}$

Papillary thyroid carcinoma (PTC) is the most common malignancy within the endocrine system. Eighty percent of PTC patients fall into a low-risk group with a 10-year cancer survival rate of $97-100 \%$, but the other $20 \%$ form a high-risk group with significant disease recurrence and morbidity. ${ }^{12,13}$ It is difficult to determine prognosis for PTC based on histopathology alone, but several prognostic factors for PTC lead to the current scoring systems. In the recent years, various molecular alterations have been used to single out malignant nodules from a pool of seemingly benign thyroid neoplasms, and $\mathrm{Ki}-67$ is one of the antigens to have been analyzed for this purpose. This study investigated whether the Ki-67 index has a significant link with several known prognostic factors for PTC. In our literature review, only a few studies had
1,2,6 Department of Surgery, UKM Medical Center, Jalan Yaakub Latiff, Kuala Lumpur, Malaysia

${ }^{3,4}$ Department of Pathology, UKM Medical Center, Jalan Yaakub Latiff, Kuala Lumpur, Malaysia

${ }^{5}$ Department of Public Health, UKM Medical Center, Jalan Yaakub Latiff, Kuala Lumpur, Malaysia

Corresponding Author: Shahrun Niza Abdullah Suhaimi, Department of Surgery, UKM Medical Center, Jalan Yaakub Latiff, Kuala Lumpur, Malaysia, Phone: +60133323773, e-mail: mrdocnan@gmail.com

How to cite this article: Suhaimi SNA, Shuhaili MA, Isa NM, et al. The Missing Link between Ki-67 and Papillary Thyroid Carcinoma. World J Endoc Surg 2020;12(2):80-85.

Source of support: Fundamental grant UKMMC

Conflict of interest: None

focused on the role of $\mathrm{Ki}-67$ as a prognostic index for PTC. Therefore, the main objective of this study was to determine the prognostic significance of Ki-67 for PTC. Furthermore, we compared Ki-67 against the metastasis, age, completeness of resection, invasion, and size (MACIS) scoring system. By correlating Ki-67 with poor prognostic features of thyroid carcinoma, we intended to predict disease recurrence among PTC patients, thus allowing for close monitoring and surveillance to improve patient outcome.

\section{Materials and Methods}

This retrospective study was conducted using 46 papillary thyroid carcinoma specimens that were retrieved from the archives of the 
Department of Pathology. These specimens were formalin-fixed and paraffin-embedded. Ethical clearance was approved by our local ethical committee (FF-2014-025). These thyroid cancers were removed during primary surgery by endocrine surgeons at UKMMC between 2006 and 2013. The surgeries were total thyroidectomies with and without central or lateral neck dissection. The diagnosis of papillary thyroid carcinoma was based on the characteristic cytological features: nuclear grooving, pseudo-inclusion, and powdery chromatin. All of these patients had undergone radioactive iodine ablation (RAl) followed by thyroxine suppressive therapy. Some of the patients had radiotherapy to the neck prior to RAl if the tumor proved to be aggressive. All patients were treated using the standard management for PTC based on international differentiated thyroid carcinoma guidelines. Thyroglobulin was monitored yearly as a marker for tumor recurrence.

From the operative and histopathology reports, important data on thyroid carcinomas were collected, including patient age and gender, tumor size, completeness of surgery, extrathyroidal extension, vascular invasion, and cervical lymph node involvement, which was unilateral, bilateral, or included in the central compartment. We also reviewed the number of lymph nodes involved and determined the ratio of the number of lymph nodes involved to the number of lymph nodes removed. Distant metastases were evidence of tumor spread beyond the neck area at the time of initial clinical presentation. Disease recurrence was calculated from 3 years after the first treatment began.

\section{Ki-67 Immunohistochemistry and Labeling Index}

We performed immunostaining using $4 \mu \mathrm{m}$ thick sections of formalin-fixed and paraffin-embedded tissue of the primary thyroid tumors. Mouse monoclonal anti-human Ki-67 Antigen Clone MIB-1 (Code No. M7240, Dako, Denmark) was used at a dilution of 1:100 as a primary antibody. Normal human tonsil tissue was used as the positive control tissue. Immunohistochemical staining was performed on the tissue sections using the protocol from the EnVision FLEX Mini Kit, High pH (Code No. K8023, Dako, Denmark). The primary antibody was diluted to the optimal concentration using Antibody Diluent, Dako REAL (Code No. S2022, Dako, Denmark). Washing steps between each reagent were performed using EnVision FLEX Wash Buffer 20× (Code No. K8007, Dako, Denmark) diluted to a $1 \times$ working solution using deionized water. The $1 \times$ DABcontaining substrate working solution was prepared by diluting the $50 \times$ concentrated EnVision FLEX DAB + Chromogen with Envision FLEX Substrate Buffer (Code No. K8023, Dako, Denmark).

The slides were incubated on hot plate at $62^{\circ} \mathrm{C}$ for 30 minutes. An initial dewaxing step was performed using $2 \times$ xylene (Merck, Germany), and the rehydration step was performed using decreasing alcohol solutions (100\%, $80 \%$, and $70 \%)$ prepared from 100\% Surgipath Reagent Alcohol (Leica Microsystems, USA). Next, the slides were rinsed in running tap water for 3 minutes. The slides were subsequently incubated with EnVision FLEX PeroxidaseBlocking Reagent (Code No. K8023, Dako, Denmark) for 5 minutes followed by rinsing with running tap water. The antigen retrieval step was performed in the DakoPTLink (Product No. PT10126, Dako, Cytomation, USA) using the EnVision FLEX Target Retrieval Solution, High pH (Code No. K8004, Dako, Denmark) at a temperature of $95^{\circ} \mathrm{C}$ for 20 minutes, followed by cooling at room temperature for 20 minutes and rinsing with running tap water.

Slides were then incubated for 20 minutes at room temperature with the primary antibody, followed by incubation with EnVision
FLEX HRP (Code No. K8023, Dako Denmark) for 20 minutes. Sections were then incubated with $1 \times$ DAB-containing Substrate Working Solution for 10 minutes. The slides were then counterstained with hematoxylin 2 (REF 7231, ThermoScientific, USA) for 10 seconds, followed by a dehydration step using increasing alcohol solutions $(80 \%, 90 \%, 100 \%$, and $100 \%)$ and $2 \times$ xylene. Finally, the slides were mounted and cover-slipped using DPX-mounting medium (Cat. No.: 100579, Merck, Milipore, Germany).

Evaluation of the Ki-67 labeling index was performed by two independent pathologists. The slides were reviewed and the Ki-67 expression immunopositivity were calculated at $40 \times$ magnification and expressed in percentages. Any discrepancies in the Ki-67 findings were reviewed by both pathologists for consensus. The control for the IHC study was sections from normal tonsils. Ki-67 was positively stained in the germinal center of the lymphoid follicles, highlighting the normal highly proliferating dark zone of the germinal center. Only nuclear staining is taken as a positive (Fig. 1). Based on Miyauchi et al., ${ }^{10}$ the Ki-67 findings were classified into three groups: group I, Ki-67 LI <5\% (low); group II, Ki-67 LI 5-10\% (intermediate); and group III, Ki-67 LI >10\% (high).

\section{Results}

\section{Patient Background and Tumor Characteristics}

A total of 46 patients were included in the study with a mean age of 47.98 years at diagnosis with $71.7 \%$ (33) were female, and $28.3 \%$ (13) were male. The majority of the patients (67\%) (31) were Malays, while the rest were primarily Chinese and Indian (Table 1).

In this study, the mean tumor size was $3 \mathrm{~cm}$, and $59 \%$ of the patients were observed to have vascular invasion and extrathyroidal extension. Distant metastases were seen in $21 \%$ of cases, and $78 \%$ had complete tumor resection during primary surgery. $\mathrm{Ki}-67$ immunostaining gave brown granular nuclear reactivity finely dispersed over the nucleus with focal denser nucleoli-like positivity (Figs 1 to 3). Ki-67 staining levels were found to be low overall among the PTC samples: $65 \%$ were in the low staining group $(<5 \%)$; $30 \%$ were in the intermediate group (5-10\%), and only $4 \%$ were in the high Ki-67 staining group (>10\%). We then calculated each patient's prognosis based on the MACIS scoring system, and $61 \%$ of the patients were found to be in the low risk group, with a $99 \%$ chance of 20 year survival, while $39 \%$ of cases were placed in the intermediate and poor prognosis groups. After 3 years of follow-up, tumor recurrences were observed in $14 \%$ of cases (Table 2).

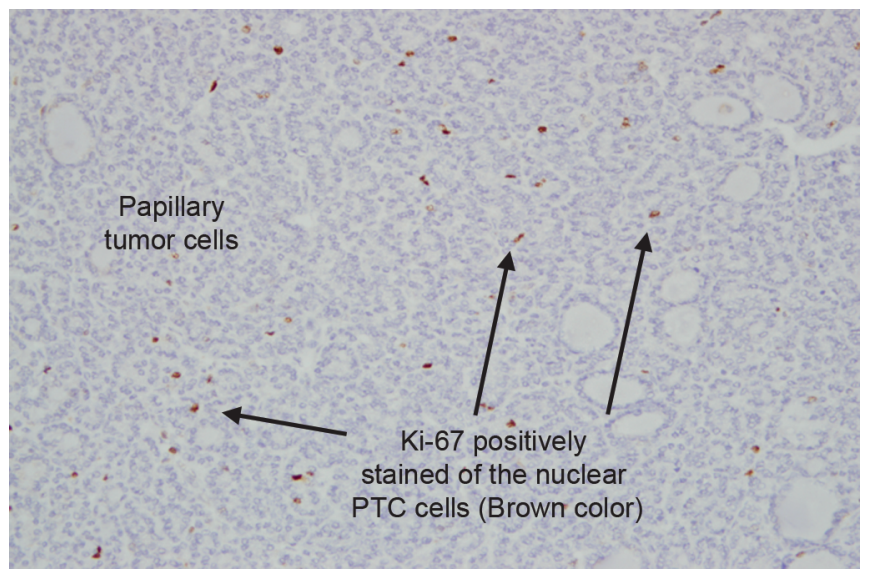

Fig. 1: Ki-67 labeling index $<5 \%$ 


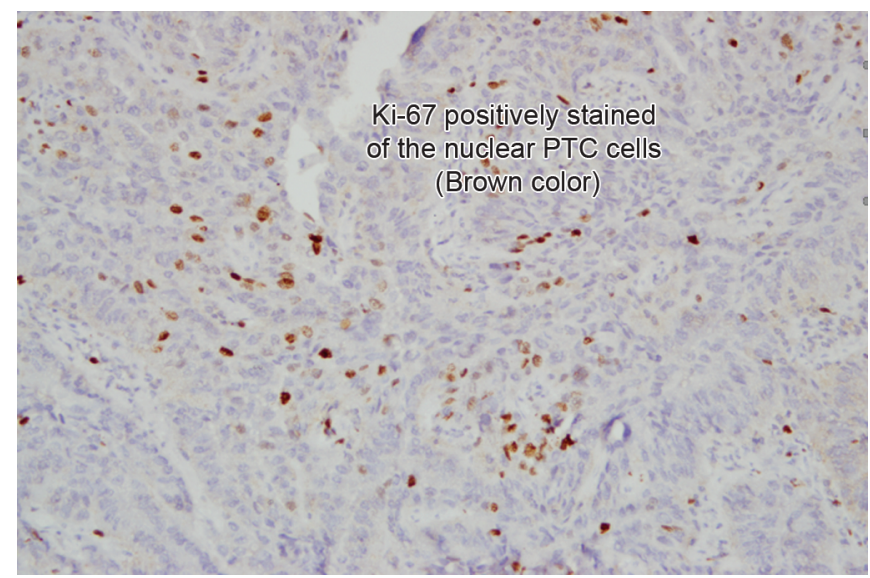

Fig. 2: Ki-67 labeling index 5-10\%

Table 1: Sociodemographic characteristics of the patients

\begin{tabular}{ll}
\hline Characteristics & Number, $n$ (\%) \\
\hline Number of patients, $n$ & 46 \\
Age (years) & \\
Gender & $49.5(33.75-61.75)$ \\
$\quad$ Male & $13(28.3)$ \\
$\quad$ Female & $33(71.7)$ \\
Ethnicity & \\
$\quad$ Malaysian & $31(67.4)$ \\
Chinese & $9(19.6)$ \\
Indian & $4(8.7)$ \\
Other & $2(4.3)$ \\
\hline
\end{tabular}

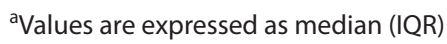

\section{Prognostic Significance of Ki-67}

There were no significant differences found for age ( $p$ value $=0.543$ ), gender $(p$ value $=0.066)$, or race $(p$ value $=0.098)$. Additionally, there were no significant differences found according to tumor size, completeness of surgery, or cervical lymph nodes involvement. However, vascular invasion, distant metastasis, and extrathyroidal extension were seen as significant predictors for Ki-67 positivity ( $p$ value $<0.05$ ). When we compared tumor recurrence at 3 years, there were 5 recurrences seen, and none of them had low Ki-67 staining. In the non-recurrence group, the majority of patient samples had low Ki-67 staining. The differences were significant ( $p$ value $<0.05$ ) (Table 3 ). Next, we further analyzed Ki-67 staining compared to the MACIS scoring system. We divided the patient samples into two groups: a lowrisk group (99\% chance of 20 year survival) and a high-risk group ( less than $90 \%$ chance of 20 year survival). A statistical significant difference was observed between these two groups. The low-risk group had $50 \%$ of low $\mathrm{Ki}-67$ unlike high-risk group to have only $7 \%$ of low Ki-67 ( $p$ value $<0.05$ ) (Table 4 ).

We then explored the relationship between $\mathrm{Ki}-67$ and tumor recurrence at 3 years. By plotting receiver-operating characteristic (ROC) curve, we deduced there was a significant connection between the two factors (Table 5). We concluded that Ki-67 is a good predictor for tumor recurrence at 3 years based on the calculated predictive values, sensitivity, and specificity (Table 5).

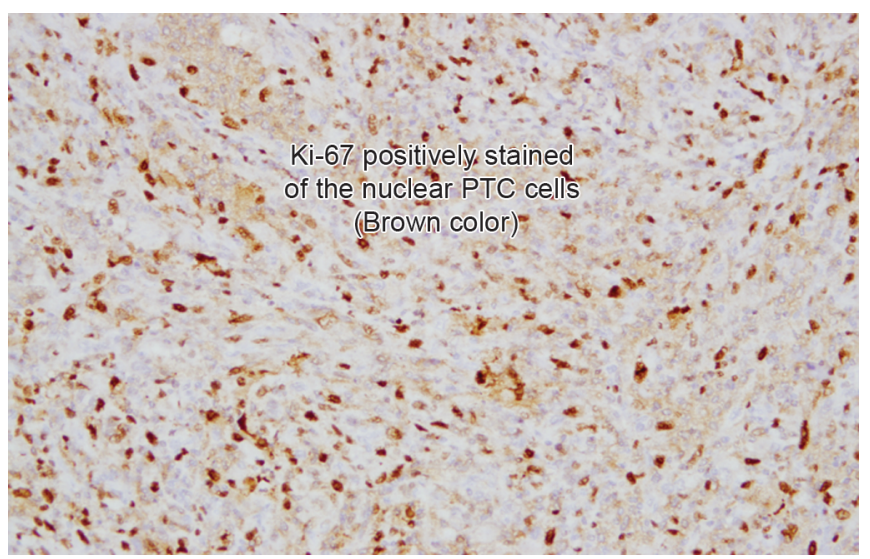

Fig. 3: Ki-67 labeling index $>10 \%$. Ki-67 positively stained of the nuclear PTC cells (Brown color)

Table 2: Characteristic features of papillary thyroid carcinoma

\begin{tabular}{lc}
\hline Specific data characteristics & Number, $n$ (\%) \\
\hline $\begin{array}{l}\text { Diameter of the tumor }(\mathrm{cm}), \\
\text { median }\end{array}$ & $2.85(1.50-3.50)$ \\
Complete resection & \\
$\quad$ Yes & $36(78.3)$ \\
No & $10(21.7)$ \\
Local vascular invasion & \\
Yes & $27(58.7)$ \\
No & $19(41.3)$ \\
Distant metastases & \\
Yes & $10(21.7)$ \\
No & $36(78.3)$ \\
Extrathyroid extension & \\
Yes & $27(58.7)$ \\
No & $19(41.3)$ \\
Prognosis (MACIS 20-year survival) & \\
$24 \%$ & $8(17.4)$ \\
$56 \%$ & $5(10.9)$ \\
$89 \%$ & $5(10.9)$ \\
$99 \%$ & $28(60.8)$ \\
Ki-67 labeling index & \\
$<5 \%$ & $30(65.2)$ \\
$5-10 \%$ & $14(30.4)$ \\
$>10 \%$ & $2(4.3)$ \\
Recurrent disease in 3 years $(n=35)$ & $5(14.3)$ \\
Yes & $30(85.7)$ \\
No & \\
\hline
\end{tabular}

\section{Discussion}

PTC is the most common cancer requiring endocrine surgery. Generally, the prognosis for PTC is excellent due to its indolent behavior, although the presence of cervical lymphadenopathy is common at initial presentation. However, mortality rates for recurrent PTC were reported up to be up to $10 \% .{ }^{14,15}$ Several clinical features, including male gender, age above 40 years, extrathyroidal extension, and distant metastasis, have been previously 
Table 3: Tumor characteristics and the Ki-67 labeling index

\begin{tabular}{|c|c|c|c|c|}
\hline \multirow{2}{*}{$\begin{array}{l}\text { Specific data } \\
\text { characteristics }\end{array}$} & \multicolumn{3}{|c|}{ Ki-67 labeling index, $n(\%)$} & \multirow[b]{2}{*}{$p$} \\
\hline & $<5 \%$ & $5-10 \%$ & $>10 \%$ & \\
\hline $\begin{array}{l}\text { Diameter of the } \\
\text { tumor (cm) } \\
\text { median (IQ). }\end{array}$ & $\begin{array}{l}2.25 \\
(1.50-3.00)\end{array}$ & $\begin{array}{l}3.50 \\
(3.00-3.50)\end{array}$ & $\begin{array}{l}2.00 \\
(1.50-2.50)\end{array}$ & 0.38 \\
\hline \multicolumn{5}{|c|}{ Complete resection $^{\mathrm{b}}$} \\
\hline Yes & $26(72.2)$ & $8(22.2)$ & $2(5.6)$ & 0.089 \\
\hline No & $4(40.0)$ & $6(60.0)$ & $0(0.0)$ & \\
\hline \multicolumn{5}{|c|}{ Local vascular invasion ${ }^{\mathrm{b}}$} \\
\hline Yes & $13(48.1)$ & $12(44.4)$ & $2(7.4)$ & $0.006^{*}$ \\
\hline No & $17(89.5)$ & $2(10.5)$ & $0(0.0)$ & \\
\hline \multicolumn{5}{|c|}{ Distant metastases ${ }^{\mathrm{b}}$} \\
\hline Yes & $3(30.0)$ & $5(50.0)$ & $2(20.0)$ & $0.005^{*}$ \\
\hline No & $27(75.0)$ & $9(25.0)$ & $0(0.0)$ & \\
\hline \multicolumn{5}{|c|}{ Extrathyroid extension ${ }^{\mathrm{b}}$} \\
\hline Yes & $13(48.2)$ & $12(44.4)$ & $2(7.4)$ & $0.006^{*}$ \\
\hline No & $17(89.5)$ & $2(10.5)$ & $0(0.0)$ & \\
\hline \multicolumn{5}{|c|}{ Recurrent disease in 3 years $(n=35)^{b}$} \\
\hline Yes & $0(0.00)$ & $4(11.4)$ & $1(2.8)$ & $0.010^{*}$ \\
\hline No & $21(62.9)$ & $8(22.9)$ & $1(2.8)$ & \\
\hline
\end{tabular}

*Significant if $(p<0.05)$

${ }^{a}$ Kruskal Wallis test (values are expressed in median, [IQR] Tukey's Hinges) ${ }^{\mathrm{b}}$ Fisher exact test

Table 4: Prognosis association between MACIS staging system (20-year survival) and the Ki-67 labeling index

\begin{tabular}{lllll}
\hline & \multicolumn{3}{c}{ Ki-67 labeling index, $n$ (\%) } & \\
\cline { 2 - 4 } Characteristics & $<5 \%$ & $5-10 \%$ & $>10 \%$ & $p$ \\
\hline MACIS staging system $(n=46)$ & & & \\
MACIS 99\% & $23(50.00)$ & $5(10.87)$ & $0(0.0)$ & $0.007^{*}$ \\
MACIS (89\%+ & $7(15.22)$ & $9(19.57)$ & $2(4.35)$ & \\
$56 \%+24 \%)$ & & & & \\
\hline
\end{tabular}

Fisher exact test

*Significant if $(p<0.05)$

reported as poor prognostic features in PTC. ${ }^{16,17}$ Therefore, several risk stratification systems have been created for prognostication purposes, such as AMES (age, metastasis, extrathyroidal extension, size), AGES (age of the patient, histologic grade of the tumor, extent of the tumor (extrathyroidal invasion or distant metastases), and size of the primary tumor EORTC (European organisation for research and treatment of cancer), and MACIS (distant metastasis, patient age, completeness of resection, local invasion, and tumor size) score. $^{13,18,19}$ These systems stratify each PTC tumor based on the aforementioned tumor characteristics, which then determines the best course of treatment for the patient.

$\mathrm{Ki}-67$ is a unique proliferative marker detected by $\mathrm{IHC}$, and its role has been implicated in several carcinomas, such as multiple myeloma, ${ }^{3}$ prostate cancer, ${ }^{4}$ soft tissue sarcma, ${ }^{5}$ and breast carcinoma. ${ }^{6,7}$ In thyroid carcinomas, $\mathrm{Ki}-67$ has been analyzed in both benign and malignant nodules, and a malignant nodule would hypothetically have a high proliferative index when compared to its benign counterpart. However, studies reported conflicting results: some supported the use of Ki-67 as a reliable biological marker, ${ }^{20}$ and other studies found no significant
Table 5: Receiver-operating characteristic (ROC) curve (a) for Ki-67 labeling index for recurrent of disease in 3 years (b)

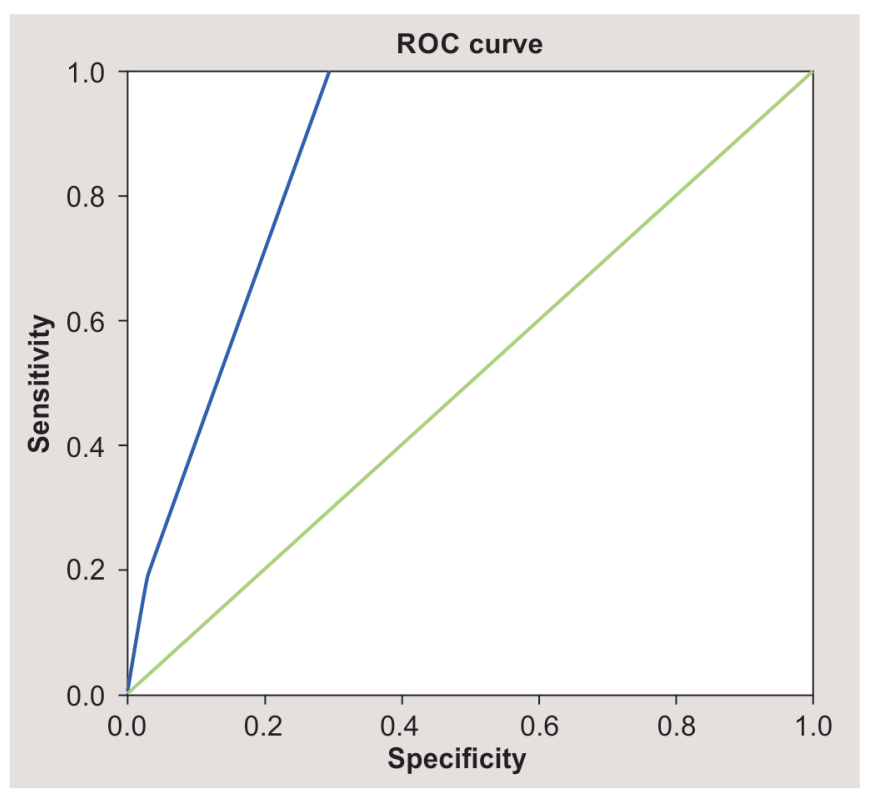

\begin{tabular}{|c|c|c|c|c|c|}
\hline \multicolumn{6}{|c|}{ Area under the curve (AUC) } \\
\hline \multirow[b]{2}{*}{ Area } & \multirow[b]{2}{*}{ Std. error ${ }^{a}$} & \multirow{2}{*}{\multicolumn{2}{|c|}{$\begin{array}{l}\text { Asymptotic } \\
\text { sig. }{ }^{b}\end{array}$}} & \multicolumn{2}{|c|}{ Asymptotic 95\% Cl } \\
\hline & & & & $\begin{array}{l}\text { Lower } \\
\text { bound }\end{array}$ & $\begin{array}{l}\text { Upper } \\
\text { bound }\end{array}$ \\
\hline 0.863 & 0.064 & 0.010 & & 0.737 & 0.990 \\
\hline \multicolumn{3}{|c|}{ Positive predictive value (PPV) } & \multicolumn{2}{|c|}{$35.7 \%$} & \\
\hline \multicolumn{3}{|c|}{ Negative predictive value (NPV) } & \multicolumn{2}{|c|}{$100 \%$} & \\
\hline \multicolumn{2}{|c|}{ Sensitivity } & \multicolumn{3}{|c|}{$100 \%$} & \\
\hline \multicolumn{2}{|c|}{ Specificity } & \multicolumn{3}{|c|}{$70 \%$} & \\
\hline
\end{tabular}

results. ${ }^{21}$ Despite those previous studies, Ki-67 has been shown to have progressively increased within multinodular goiters that transformed into several cancers, including follicular adenoma, papillary carcinoma, follicular carcinoma, and medullary carcinoma. The highest Ki-67 percentages were seen in poorly differentiated thyroid carcinoma ${ }^{9,22}$ and anaplastic thyroid carcinoma. To date, there have been few studies that focused on the prognostic factors of PTC and Ki-67.

In the current study, we investigated the connection between Ki-67 and several prognostic factors for PTC. The prognostic factors included extrathyroidal extension, vascular invasion, distant metastasis, cervical lymph nodes involvement, complete tumor removal during initial surgery, and disease recurrence at 3 year follow-up. Tumor extension beyond the thyroid capsule is generally associated with poor prognosis, and various multivariate analyses have supported this conclusion..$^{19,23}$ In a Greek study, using retrospective analysis of 832 patients with PTC, tumor extension combined with other prognostic factors significantly decreased the survival rate. ${ }^{24}$ Vascular invasion was also a sign of tumor aggressiveness that led to distant metastasis via a hematogenous route. ${ }^{25}$ Based on statistical analysis, we observed that vascular invasion, distant metastasis, extrathyroidal extension, and disease recurrence at 3 years were significantly related to Ki-67 levels. 
The findings here are similar to reports by Ito et al. ${ }^{10,26}$ Those studies indicated that tumors with a high proliferative index have a high proclivity for invading adjacent structures such as the trachea, surrounding muscle, and jugular veins, and for metastasizing to distant organs.

Cervical lymphadenopathy is also common at presentation in PTC. However, the influence of cervical lymph node at initial presentation has been controversial and was previously shown not to influence survival rate. ${ }^{27}$ Our research revealed that $\mathrm{Ki}-67$ had no significant link to cervical lymph nodes' involvement, which was in agreement with previous reports. ${ }^{26} \mathrm{It}$ is postulated that the metastatic activity within the cervical lymph nodes is not related to the proliferative activity within the tumor. ${ }^{28}$ Tumor size is also an important aspect of tumor morphology. The risk of recurrence and tumor-related death is proportional to the increased size of the primary tumor. ${ }^{14,29}$ Equally, age has been considered an important prognostic factor for PTC, with 40 year old as the cutoff point for cancer survival; mortality increases progressively after the age of $40 .^{30,31}$ Several studies reported that Ki-67 had significant correlation with tumor size and increasing age. ${ }^{28,32,33}$ For example, Yorukoglu et al., ${ }^{34}$ in their study of $\mathrm{Ki}-67$ expression in papillary thyroid carcinoma, showed the $\mathrm{Ki}-67$ proliferation index to be significantly low for micropapillary carcinoma (1\%) compared to papillary carcinoma (4\%). However, there were no differences between papillary carcinoma variants. Our study showed no significance related to tumor size or patient age. Our study included only tumors larger than $1 \mathrm{~cm}$; so, micropapillary carcinoma was excluded from the study. We believe that for tumors, other than micropapillary, there would not be any difference in Ki-67 levels, unless the tumor involved was either poorly differentiated or an anaplastic carcinoma. ${ }^{28}$

Currently, there are 14 available staging systems that can be used to predict cancer survival in patients with PTC, irrespective of the histologic type. We chose the MACIS system because it is the most predictive staging system and should be the staging system of choice. ${ }^{13}$ Therefore, we calculated the prognosis for all PTC patients using the MACIS scoring system for 20 year survival rates, and we compared the results with Ki-67 levels. We matched up the good prognosis group ( $99 \%$ chance of 20 year survival) with the remaining group $(89 \%+56 \%+24 \%$ chance of 20 year survival). $\mathrm{Ki}-67$ is significantly lower in the good prognosis group, indicating that this group had a low proliferative index. This result could be expected because PTC itself is an indolent tumor with an excellent overall survival rate. In our study, Ki-67 predicts tumor recurrent at 3 years at $86 \%$ with high sensitivity and specificity. Therefore, we concluded that $\mathrm{Ki}-67$ analysis provided a good diagnostic tool for predicting the recurrence of PTC.

A further inquiry arose from this study: Ki-67 may further substantiate PTCs that fall under the categories of "intermediate" or "worse" prognosis. We believe that for the PTCs at risk of an increase in $\mathrm{Ki}-67$, the patients should have frequent monitoring through yearly follow-up thyroglobulin monitoring, along with prolonged thyroxine suppressive therapy. However, we do not recommend $\mathrm{Ki}-67$ analysis for the so-called good prognosis tumors because it will not be cost effective. In addition, using Ki- 67 as a sole prognostication tool in PTCs is not recommended. The prognosis of PTC must be considered based on clinical presentation, tumor behavior, stage, and the final histopathology report. Therefore, $\mathrm{Ki}-67$ analysis acts as additional information for clinicians in the face of aggressive PTCs. Currently, there are other markers that evaluate cell proliferation activity. For example, mitotic figure count (MFC) on hematoxylin and eosin-stained tissue, or proliferating cell nuclear antigen (PCNA), are other markers being used. However, both MFC and PCNA showed less reliable results than Ki-67 itself. ${ }^{14,35}$

We acknowledge that this study has limitations, such as a small sample size, and a short study duration. By having a larger sample size, we could use multivariate and log regression analysis to further solidify the results. As with any immunohistochemical examination, interobserver variability is also a concern for result reproducibility. However, a previous study showed that for Ki-67 analysis, interobserver variability was negligible, as long as two experienced pathologists performed the tumor analyses. ${ }^{36,37}$ Our pathologists were well trained in the interpretation of Ki-67; therefore, we believe our data to have high accuracy and reproducibility. Based on the findings of Miyauchi et al., ${ }^{10}$ we find this classification system was simpler and more practical for this study. We noted that Ki-67-positive staining was generally low in PTC, similar to other reported findings. ${ }^{28,34}$ These results were probably due to the indolent nature of this carcinoma.

\section{Conclusion}

Ki-67 proliferative index correlates well with several poor prognostic features, such as extrathyroidal extension, vascular invasion, and distant metastasis. Ki-67 was proven to be lower among good prognosis PTCs, and Ki-67 was shown to be a good diagnostic tool to predict recurrence of PTC

\section{References}

1. Gerdes J, Lemke H, Baisch $\mathrm{H}$, et al. Cell cycle analysis of a cell proliferation associated human nuclear antigen defined by the monoclonal antibody Ki-67. J Immunol 1984;133(4):1710-1715.

2. Gerdes J, Schwab U, Lemke $\mathrm{H}$, et al. Production of a mouse monoclonal antibody reactive with a human nuclear antigen associated with cell proliferation. Int J Cancer 1983;31(1):13-20. DOI: 10.1002/ijc.2910310104.

3. Drach J, Gattringer C, Glassl H, et al. The biological and clinical significance of the $\mathrm{Ki}-67$ growth fraction in multiple myeloma. Hematol Oncol 1992;10(2):125-134. DOI: 10.1002/hon.2900100209.

4. Borre M, Bentzen SM, Nerstrom B, et al. Tumour cell proliferation and survival in patients with prostate cancer followed expectantly. J Urol 1998;159(5):1609-1614. DOI: 10.1097/00005392-199805000-00054.

5. Huuhtanen $\mathrm{RL}$, Blomqvist $\mathrm{CP}$, Wiklund $\mathrm{TA}$, et al. Comparison of the $\mathrm{Ki}-67$ score and S-phase fraction as prognostic variables in soft tissue sarcoma. Br J Cancer 1999;79(5-6):945-951. DOI: 10.1038/ sj.bjc.6690151.

6. Rudolph P, Olsson H, Bonatz G, et al. Correlation between p5, c-erbB-2, and topoisomerase lla expression, DNA ploidy, hormonal receptor status and proliferation in 356 node-negative breast carcinomas: prognostic implications. J Pathol 1999;187(2):207-216. DOI: 10.1002/ (SICI)1096-9896(199901)187:2<207::AID-PATH223>3.0.CO;2-U.

7. Jansen RL, Hupperets PS, Arends JW, et al. MIB-1 labelling index is an independent prognostic marker in primary breast cancer. $\mathrm{Br} \mathrm{J}$ Cancer 1998;78(4):460-465. DOI: 10.1038/bjc.1998.515.

8. Aiad HA, Bashandy MA, Abdou AG, et al. Significance of AgNORs and Ki-67 proliferative markers in differential diagnosis of thyroid lesions. Pathol Oncol Res 2013;19(2):167-175. DOI: 10.1007/s12253-012-9565-1.

9. Pujani M, Arora B, Singh SK, et al. Role of Ki-67 as a proliferative marker in lesions of thyroid. Indian J Cancer 2010;47(3):304-307. DOI: 10.4103/0019-509X.64727.

10. Miyauchi A, Kudo T, Hirokawa M, et al. Ki-67 labelling index is a predictor of postoperative persistent disease and cancer growth and 
a prognostic indicator in papillary thyroid carcinoma. Eur Thyroid J 2013;2(1):57-64. DOI: 10.1159/000347148.

11. Tisell LE, Oden A, Muth A, et al. The Ki- 67 index is a prognostic marker in medullary thyroid carcinoma. Br J Cancer 2003;89(11):2093-2097. DOI: $10.1038 /$ sj.bjc.6601453.

12. Hay ID, Thompson GB, Grant CS, et al. Papillary thyroid carcinoma managed at the Mayo clinic during six decades (1940-1999): temporal trends in initial therapy and long-term outcome in 2444 consecutively treated patients. World J Surg 2002;26(8):879-885. DOI: 10.1007/ s00268-002-6612-1.

13. Lang BH, Lo CY, Chan WF, et al. Staging systems for papillary thyroid carcinoma: a review and comparison. Ann Surg 2007;245(3):366-378. DOI: 10.1097/01.sla.0000250445.92336.2a.

14. Mazzaferri EL, Kloos RT. Current approaches to primary therapy for papillary and follicular thyroid cancer. J Clin Endocrinol Metab 2001;86(4):1447-1462. DOI: 10.1210/jcem.86.4.7407.

15. Eustatia-Rutten CF, Corssmit EP, Biermasz NR, et al. Survival and death causes in differentiated thyroid carcinoma. J Clin Endocrinol Metab 2006;91(1):313-319. DOI: 10.1210/jc.2005-1322.

16. Ito $Y$, Tomoda $C$, Uruno $T$, et al. Prognostic significance of extrathyroidal extension of papillary thyroid carcinoma: massive but not minimal extension affects the relapse-free survival. World J Surg 2006;30(5):780-786. DOI: 10.1007/s00268-005-0270-z.

17. Ito $Y$, Tomoda C, Urona $T$, et al. Ultrasound-detectable and anatomopathologically detectable node metastasis in the lateral compartment as indicators of worse relapse-free survival in patients with papillary thyroid carcinoma. World J Surg 2005;29(7):917-920. DOI: 10.1007/s00268-005-7789-x.

18. Cady B, Rosai R. An expanded view of risk group definition in differentiated thyroid carcinoma. Surgery 1988;104(6):947-953. DOI: 10.1016/S0039-6060(98)70034-0.

19. Hay ID, Bergstrahl EJ, Goellner JR, et al. Predicting outcome in papillary thyroid carcinoma: development of a reliable prognostic scoring system in a cohort of 1,779 patients surgically treated at one institution during 1940 through 1989. Surgery 1993;114(6): 1050-1058.

20. Saiz AD, Olvera $M$, Rezk S, et al. Immunohistochemical expression of cyclin D1, E2F-1 and Ki-67 in benign and malignant thyroid lesions. J Pathol 2002;198(2):157-162. DOI: 10.1002/path.1185.

21. Song $Q$, Wang $D$, Lou $Y$, et al. Diagnostic significance of CK19, TG, Ki67 and galectin-3 expression for papillary thyroid carcinoma in the northeastern region of China. Diagn Pathol 2011;6:126. DOI: 10.1186/1746-1596-6-126.

22. Erickson LA, Jin L, Wollan PC, et al. Expression of p27 kip 1 and ki 67 in benign and malignant thyroid tumors. Mod Pathol 1998;11(2):169-174.

23. Shaha AR, Loree TR, Shah JP. Prognostic factors and risk group analysis in follicular carcinoma of the thyroid. Surgery 1995;118(6):1136-1138. DOI: 10.1016/S0039-6060(05)80124-2.
24. Tzavara I, Vlassopoulou B, Alevizaki C, et al. Differentiated thyroid cancer: a retrospective analysis of 832 cases from Greece. Clin Endocrinol (Oxf) 1999;50(5):643-654. DOI: 10.1046/j.13652265.1999.00715.x.

25. Falvo L, Catania A, D'Andrea V, et al. Prognostic importance of histologic vascular invasion in papillary thyroid carcinoma. Ann Surg 2005;241(4):640-646. DOI: 10.1097/01.sla.0000157317.60536.08.

26. Ito $Y$, Akira M, Kakudo K, et al. Prognostic significance of Ki- 67 labelling index in papillary thyroid carcinoma. World J Surg 2010;34(12): 3015-3021. DOI: 10.1007/s00268-010-0746-3.

27. Lin JD, Liou MJ, Chao TC, et al. Prognostic variables of papillary and follicular thyroid carcinoma patients with lymph nodes metastases and without distant metastases. Endocr Relat Cancer 1999;6(1): 109-115. DOI: 10.1677/erc.0.0060109.

28. Kjellman P, Wallin G, Hoog A, et al. MIB-1 index in thyroid tumors: a predictor of the clinical course in papillary thyroid carcinoma. Thyroid 2003;13(4):371-380. DOI: 10.1089/105072503321669866.

29. Schlumberger M, Pacici F. Thyroid tumors. 2nd ed., Nucleon; 2003. p. 111-125.

30. Cunningham MP, Duda RB, Recant W, et al. Survival discriminants for differentiated thyroid cancer. Am J Surg 1990;160(4):344-347. DOI: 10.1016/S0002-9610(05)80539-2.

31. Ward LS, Assumpcao LV. Thyroid cancer: prognostic factors and treatment. Arq Bras Endocrinol Metabol 2004;48(1):126-136. DOI: 10.1590/S0004-27302004000100014.

32. Lantsov D, Meirmanov S, Nakashima M, et al. Cyclin D1 overexpression in thyroid papillary microcarcinoma: its association with tumour size and aberrant beta-cathenin expression. Histopathology 2005;47(3):248-256. DOI: 10.1111/j.1365-2559.2005.02218.x.

33. Siironen $P$, Nordling $S$, Louhimo J, et al. Immunohistochemical expression of $\mathrm{BCl}-2, \mathrm{Ki}-67$ and $\mathrm{p} 21$ in patients with papillary thyroid cancer. Tumour Biol 2005;26(1):50-56. DOI: 10.1159/000084340.

34. Yorukoglu A, Yalcin N, Avci A, et al. Significance of IMP3, nucleoplasmin and Ki-67 expression in papillary thyroid carcinoma. Int J Surg Pathol 2015;23(1):5-12. DOI: 10.1177/1066896914554832.

35. Sullivan RP, Mortimer G, Murschertaigh IO. Cell proliferation in breast tumors: analysis of histological parameters Ki-67 and PCNA expression. Ir J Med Sci 1993;162(9):343-347. DOI: 10.1007/ BF02942162.

36. Gunia S, Albrescht K, Koch S, et al. Ki-67 staining index and neuroendocrine differentiation aggravate adverse prognostic parameters in prostate cancer and are characterized by negligible inter-observer variability. World J Urol 2008;26(3):243-250. DOI: 10.1007/s00345-008-0257-0.

37. Presusser $M$, Heinzi $H$, Gelpi $E$, et al. Ki-67 index in intracranial ependymoma: a promising histopathological candidate biomarker. Histopathology 2008;53(1):39-47. DOI: 10.1111/j.13652559.2008.03065.x. 